\title{
ON THE QUASIREGULARITY OF A LIMIT MAPPING
}

\author{
PETER LINDQVIST
}

\section{Introduction}

A well-known theorem of Weierstrass states that the limit function for a convergent sequence of analytic functions is analytic. An analogue for quasiregular mappings was obtained in 1967 by Reshetnyak [3]:

1.1. Theorem. Suppose that the mappings $f^{v}$ are $K_{v}$-quasiregular in a domain $G$ in $\boldsymbol{R}^{n}, v=1,2,3, \ldots$ If $\lim f^{v}=f$ uniformly in compact subsets of $G$ and if $\lim \inf K_{v}$ $<\infty$, then the limit function fitself is $K$-quasiregular with the dilatation $K \leqq \lim \inf K_{v}$.

The crucial step in connection with this convergence phenomenon is, in a sense, the weak convergence of the Jacobian determinants $J\left(f^{1}\right), J\left(f^{2}\right), J\left(f^{3}\right), \ldots$, i.e. the validity of

$$
\lim _{v \rightarrow \infty} \int_{Q} J\left(f^{v}\right) d m=\int_{Q} J(f) d m
$$

whenever $Q$ is a cube with $\bar{Q} \subset \subset G$. To establish (1.2) Reshetnyak used differential forms. See also [4, II $\S 4]$.

A first step towards (1.2) is any bound of the kind expressed by Mikljukov's inequality (3.2). This consequence of quasiregularity is usually proved via the fundamental fact that quasiregular mappings are free extremals for certain variational integrals; cf. [3].

Since Theorem 1.1 in itself is not very deep, we are going to present a simple proof, avoiding both the advanced theory of differential forms and the calculus of variations. Our proof variant is essentially based only on the following fundamental property of the Jacobian determinant:

1.3. Lemma. If $g=\left(g_{1}, \ldots, g_{n}\right)$ belongs to $C(G) \cap W_{n, \text { loc }}^{1}(G)$, then

$$
\int_{G} J\left(g_{1}, \ldots, g_{j-1}, \zeta g_{j}, g_{j+1}, \ldots, g_{n}\right) d m=0 \quad(j=1,2, \ldots, n)
$$

whenever $\zeta \in C_{0}^{\infty}(G)$, i.e. $\zeta$ is smooth and has compact support in $G$. 
Equality (1.4) follows from Stokes's elementary theorem. Our method of proof is based on the recursive use of

$$
\int_{G} \zeta J(g) d m=-\int_{G} g_{j} J\left(g_{1}, \ldots, g_{j-1}, \zeta, g_{j+1}, \ldots, g_{n}\right) d m,
$$

$j=1,2, \ldots, n, \zeta \in C_{0}^{\infty}(G)$.

\section{Quasiregular mappings}

Throughout this paper, let $G$ denote a domain in $n$-dimensional space $\boldsymbol{R}^{n}, n \geqq 2$. A mapping $f=\left(f_{1}, \ldots, f_{n}\right): G \rightarrow R^{n}$ is called $K$-quasiregular in $G$ if

$1^{\circ} f \in C(G)$, i.e., $f$ is continuous in $G$,

$2^{\circ} f \in W_{n \text {, loc }}^{1}(G)$, i.e., the generalized partial derivatives $\partial f_{j} / \partial x_{k}, j, k=1, \ldots, n$, exist and are locally $n$-summable in $G$, and

$3^{\circ}$ the inequality

$$
|D f|^{n} \leqq K J(f)
$$

holds a.e. in $G$ with some constant $K<\infty$. Here

is a norm and

$$
|D f|=\left\{\Sigma\left(\frac{\partial f_{j}}{\partial x_{k}}\right)^{2}\right\}^{1 / 2}
$$

is Jacobi's determinant.

$$
J(f)=J\left(f_{1}, \ldots, f_{n}\right)
$$

For any mapping $g: G \rightarrow R^{n}$ belonging to Sobolev's space $W_{n, \text { loc }}^{1}(G)$ the inequality

$$
n^{n / 2} J(g) \leqq|D g|^{n}
$$

is valid a.e. in $G$. (The smallest number $K \geqq n^{n / 2}$ for which (2.1) holds is here called the dilatation of $f$.)

For more details we refer the reader to [1], [2], and [4]. However, no theory concerning quasiregular mappings is needed in this paper.

\section{Mikljukov's inequality}

A suitable uniform bound for the integrals $\int\left|D f^{v}\right|^{n} d m, v=1,2, \ldots$, is easily derived via Mikljukov's inequality.

3.1. Lemma (Mikljukov). Suppose that $f$ is $K$-quasiregular in $G$. Then

$$
\int_{G} \varphi^{n} J(f) d m \leqq n^{n} K^{n-1} \int_{G}|f|^{n}|\nabla \varphi|^{n} d m
$$

for each non-negative $\varphi \in C_{0}^{\infty}(G)$. 
Proof. The choice $\zeta=\varphi^{n}$ in $\left(1.4^{\prime}\right)$ yields

$$
\int_{G} \varphi^{n} J(f) d m=-n \int_{G} \varphi^{n-1} f_{1} J\left(\varphi, f_{2}, \ldots, f_{n}\right) d m,
$$

where $f=\left(f_{1}, \ldots, f_{n}\right)$. By Hadamard's inequality

$$
\left|J\left(\varphi, f_{2}, \ldots, f_{n}\right)\right| \leqq|\nabla \varphi||D f|^{n-1}
$$

a.e. in $G$. Thus Hölder's inequality and (3.3) yield

$$
\int_{G} \varphi^{n} J(f) d m \leqq n\left(\int_{G}\left|f_{1}\right|^{n}|\nabla \varphi|^{n} d m\right)^{1 / n}\left(\int_{G} \varphi^{n}|D f|^{n} d m\right)^{(n-1) / n} .
$$

Since $\left|f_{1}\right|^{n} \leqq|f|^{n}$ and

$$
\int_{G} \varphi^{n}|D f|^{n} d m \leqq K \int_{G} \varphi^{n} J(f) d m
$$

by (2.1), we arrive at (3.2) after some obvious simplifications.

\section{Weak convergence for the Jacobian determinants}

Suppose now that $f^{v}=\left(f_{1}^{v}, \ldots, f_{n}^{v}\right)$ is $K_{v}$-quasiregular in $G, v=1,2, \ldots$, and $\lim \inf K_{v}<\infty$. Suppose that the numbers $K_{v}$ converge to their limes inferior. Furthermore, assume $\lim f^{v}=f$ locally uniformly in $G$. Hence $f$ is continuous in $G$. We claim that $f$ actually belongs to Sobolev's space $W_{n, \text { loc }}^{1}(G)$. For every cube $Q, \bar{Q} \subset \subset G$, there is a corresponding constant $M_{Q}<\infty$ such that

$$
\int_{Q}\left|D f^{v}\right|^{n} d m<M_{\mathcal{Q}} \quad(v=1,2, \ldots) .
$$

This bound follows from (2.1) combined with Mikljukov's inequality (3.2), where we choose $\varphi \in C_{0}^{\infty}(G)$ so that $\varphi \mid Q=1$ and $0 \leqq \varphi \leqq 1$. By standard reasoning we obtain the local result $f \in W_{n}^{1}(Q)$. Since $Q$ was arbitrary, we have $f \in W_{n, \text { loc }}^{1}(G)$. Thus $J(f)$ is defined a.e. in $G$. Note that $J(f)$ is locally integrable over $G$.

Since we aim at obtaining Theorem 1.1 as directly as possible, we are satisfied with a slightly weaker version of (1.2); cf. Remark 4.5.

4.2. Lemma. If $\varphi \in C_{0}^{\infty}(G)$, then

$$
\lim _{v \rightarrow \infty} \int_{G} \varphi^{n} J\left(f^{v}\right) d m=\int_{G} \varphi^{n} J(f) d m .
$$

Proof. We claim that

$$
\begin{aligned}
& \lim _{v \rightarrow \infty} \int_{G} \varphi^{j-1} J\left(f_{1}^{v}, \ldots, f_{j-1}^{v}, g_{j}, \ldots, g_{n}\right) d m \\
& =\int_{G} \varphi^{j-1} J\left(f_{1}, \ldots, f_{j-1}, g_{j}, \ldots, g_{n}\right) d m
\end{aligned}
$$

for any $\varphi \in C_{0}(G)$ and for any continuous functions $g_{j}, \ldots, \mathrm{g}_{n}$ in $W_{n, \mathrm{loc}}^{1}(G) ; j=$ 
$=2,3, \ldots, n+1$. Note that (4.4) immediately implies

$$
\begin{gathered}
\lim _{v \rightarrow \infty} \int_{G} \varphi^{j-1} f_{j}^{v} J\left(f_{1}^{v}, \ldots, f_{j-1}^{v}, g_{j}, \ldots, g_{n}\right) d m \\
=\int_{G} \varphi^{j-1} f_{j} J\left(f_{1}, \ldots, f_{j-1}, g_{j}, \ldots, g_{n}\right) d m
\end{gathered}
$$

with the same $\varphi$ and $g_{j}, \ldots, g_{n}$; cf. (2.1), (2.2), and (3.2).

Let us prove (4.4) by induction with respect to $j$. For $j=2$ we have by $\left(1.4^{\prime}\right)$

$$
\int_{G} \varphi J\left(f_{1}^{v}, g_{2}, \ldots, g_{n}\right) d m=-\int_{G} f_{1}^{v} J\left(\varphi, g_{2}, \ldots, g_{n}\right) d m
$$

for any $\varphi \in C_{0}^{\infty}(G)$. The limit of the right-hand side is obvious and so $\left(1.4^{\prime}\right)$ used again yields (4.4) for any $\varphi \in C_{0}^{\infty}(G)$ when $j=2$. Observe that $C_{0}^{\infty}(G)$ is dense in $C_{0}(G)$.

Suppose now that (4.4), and, consequently, also (4.4') hold for some index $j$ in the range $2,3, \ldots, n$. If $\varphi \in C_{0}^{\infty}(G),\left(1.4^{\prime}\right)$ implies that

$$
\begin{gathered}
\int_{G} \varphi^{j} J\left(f_{1}^{v}, \ldots, f_{j}^{v}, g_{j+1}, \ldots, g_{n}\right) d m \\
=-j \int_{G} \varphi^{j-1} f_{j}^{v} J\left(f_{1}^{v}, \ldots, f_{j-1}^{v}, \varphi, g_{j+1}, \ldots, g_{n}\right) d m,
\end{gathered}
$$

and so (4.4') and (1.4') yield (4.4) with $j-1$ replaced by $j$. Observe again that $C_{0}^{\infty}(G)$ is dense in $C_{0}(G)$.

At the final step $j=n+1$ we obtain (4.3). This concludes our proof.

4.5. Remark. $1^{\circ}$ Minor technical modifications in the previous proof yield (4.3) with $\varphi^{n}$ replaced by any $\varphi$ in $C_{0}(G)$, and then some standard arguments give (1.2). However, such "improvements" are not essential for Theorem 1.1.

$2^{\circ}$ Actually, the quasiregularity of the mappings $f^{v}$ was used only to obtain the uniform bound (4.1). So the following general result is easily available:

Let $f^{v}: G \rightarrow R^{n}, v=1,2, \ldots$, be any sequence in $C(G) \cap W_{n, \text { loc }}^{1}(G)$ such that the uniform bound (4.1) holds for all cubes $\bar{Q} \subset \subset G$. If $\lim f^{v}=f$ locally uniformly in $G$, then (1.2) is valid. See [6, p. 94.]

\section{The limit theorem}

For the sake of completeness we reproduce the well-known proof for Theorem 1.1. We have to show that (2.1) holds a.e. in $G$. Fix therefore any cube $Q, \bar{Q} \subset \subset G$. Let $\varphi$ be any function in $C_{0}^{\infty}(G)$ so that $\varphi \mid Q=1,0 \leqq \varphi \leqq 1$. By convexity

$$
\int_{Q}\left|D f^{v}\right|^{n} d m \geqq \int_{Q}|D f|^{n} d m+n \sum_{j=1}^{n} \int_{Q}|D f|^{n-2} \nabla f_{j} \cdot \nabla\left(f_{j}^{v}-f_{j}\right) d m
$$

and via a suitable weakly convergent subsequence we get

$$
\int_{\boldsymbol{Q}}|D f|^{n} d m \leqq \lim _{v \rightarrow \infty} \int_{\boldsymbol{Q}}\left|D f^{v}\right|^{n} d m
$$


Here the uniform bound (4.1) plays a central rôle. Now

$$
\begin{gathered}
\varliminf_{v \rightarrow \infty} \int_{Q}\left|D f^{v}\right|^{n} d m \leqq \varliminf_{v \rightarrow \infty} \int_{G} \varphi^{n}\left|D f^{v}\right|^{n} d m \\
\leqq \varliminf_{v \rightarrow \infty} K_{v} \int_{G} \varphi^{n} J\left(f^{v}\right) d m \leqq \varliminf_{v \rightarrow \infty} K_{v} \int_{G} \varphi^{n} J(f) d m,
\end{gathered}
$$

according to (4.3). Combining (5.1) and (5.2) we arrive at

$$
\int_{Q}|D f|^{n} d m \leqq \varliminf_{v \rightarrow \infty} K_{v} \int_{G} \varphi^{n} J(f) d m .
$$

Since $\varphi$ was arbitrary and the integral is absolutely continuous, we have

$$
\int_{Q}|D f|^{n} d m \leqq \varliminf_{v \rightarrow \infty} K_{v} \int_{Q} J(f) d m
$$

for any cube $Q, \bar{Q} \subset \subset G$. This means that (2.1) holds at least with $K=\lim \inf K_{v}$. Thus $f$ is $K$-quasiregular in $G$.

\title{
References
}

[1] GRANLUnd, S., P. LindQvist, and O. MARTIO: Conformally invariant variational integrals. - Trans. Amer. Math. Soc. 277, 1983, 43-73.

[2] Martio, O., S. RICKMAN, and J. VÄISÄLÄ: Definitions for quasiregular mappings. - Ann. Acad. Sci. Fenn. Ser. A I Math. 448, 1969, $1-40$.

[3] ReshetNYAK, YU. G.: Mappings with bounded deformation as extremals of Dirichlet type integrals - Sibirsk. Mat. Zh. 9, 1968, 652-666 (Russian).

[4] Решетняк, Ю.: Простраственние Отображенения с Ограниченным Искажением. - Изд. «НАУКА», Новосибирск, 1982.

\author{
Helsinki University of Technology \\ Institute of Mathematics \\ SF-02150 Espoo \\ Finland
}

Received 18 January 1984 